\title{
Purely Endoscopic Repair of Traumatic Cerebrospinal Fluid Rhinorrhea From the Anterior Skull Base -Case Report-
}

\author{
Mika Komatsu, ${ }^{1}$ Fuminari KOMATsu, ${ }^{1}$ Luigi M. CAVAllo, ${ }^{2}$ Domenico SOlaRI, ${ }^{2}$ \\ Vita STAGnO, ${ }^{2}$ Tooru INOUE, ${ }^{1}$ and Paolo CAPPABIANCA ${ }^{2}$ \\ ${ }^{1}$ Department of Neurosurgery, Faculty of Medicine, Fukuoka University, Fukuoka; \\ ${ }^{2}$ Department of Neurological Sciences, Division of Neurosurgery, \\ Università degli Studi di Napoli Federico II, Naples, Italy
}

\begin{abstract}
A 38-year-old woman presented with a case of post-traumatic ethmoidal cerebrospinal fluid (CSF) leak that was repaired using a purely endoscopic endonasal approach. Six weeks after a mild domestic maxillofacial trauma, she started complaining of clear, watery discharge from the left nostril and headache. Neuroimaging investigations disclosed a linear fracture of the left anterior ethmoidal roof without evidence of large bony breaches or any brain tissue damage. After conservative medical treatment with carbonic anhydrase inhibitors failed, she was referred to our hospital for surgical repair of the osteodural defect. An endoscopic endonasal transethmoidal approach was successfully performed, and an overlay technique was used to reconstruct the defect. The patient was discharged without clinical evidence of CSF rhinorrhea and no leaks were apparent at the 3-month follow-up clinical and radiological examinations. Post-traumatic CSF rhinorrhea occurs in cases of dural tears associated with small bone breaks, most frequently involving the anterior skull base. Recovery is often spontaneous following only bed rest, or with medical treatments such as inhibitors of carbonic anhydrase diuretics, steroids, or eventually stool softeners to help reduce CSF pressure. Surgical repair is required when first-line conservative treatment proves ineffective. The present case shows that the endoscopic endonasal technique for the management of CSF leaks provides a less invasive surgical route to achieve valid dural repair.
\end{abstract}

Key words: maxillofacial trauma, cerebrospinal fluid rhinorrhea, anterior skull base fracture, endoscopic endonasal approach, cerebrospinal fluid leak

\section{Introduction}

Cerebrospinal fluid (CSF) leak is a medical condition involving abnormal release of CSF from the subarachnoid space into an extracranial compartment and originating from a breakdown of a physiological barrier, i.e., from either osseous or dural defects. Such leaks can be spontaneous or traumatic, and frequently result after disruptive craniofacial trauma. The anatomical regions that represent the most common sites of origin for CSF leaks after anterior skull-base trauma are the cribriform plate, the roof of the sphenoid sinus, and the posterior wall of the frontal sinus, since the dura mater is strictly adherent to the bone at these sites.

Minor head injuries and blunt craniofacial traumas can sometimes result in small fractures that, although remaining undetected on first-line x-ray screening, can cause CSF rhinorrhea. The majority of cases respond to conservative treatment (bed rest with slight reverse-Trendelenburg position, medical treatment, with or without place-

Received April 8, 2010;

Accepted August 2, 2010 ment of external lumbar drainage), but surgical repair is required in patients who appear refractory to such therapies. Both transcranial and transnasal approaches have been adopted for the surgical treatment of post-traumatic rhinorrhea. ${ }^{18,20,22)}$ The widespread application of endoscopic endonasal techniques extending beyond the sellar region ${ }^{2,4,11)}$ has recently led neurosurgeons and otorhinolaryngological surgeons to advocate this technique as a possible treatment option for CSF leaks..$^{5,6,15,18,22)}$

We report a case of traumatic ethmoidal leak that was successfully treated using a purely endoscopic endonasal method.

\section{Case Report}

A 38-year-old woman experienced a mild craniofacial trauma in a domestic accident, and 6 weeks later started complaining of sub-continuous frontal headache, at the same time describing a clear, watery discharge from the left nostril. Although a biochemical assay for $\beta_{2}$-transferrin evaluation yielded negative results, post-traumatic CSF leak was suspected. The patient was observed during a 

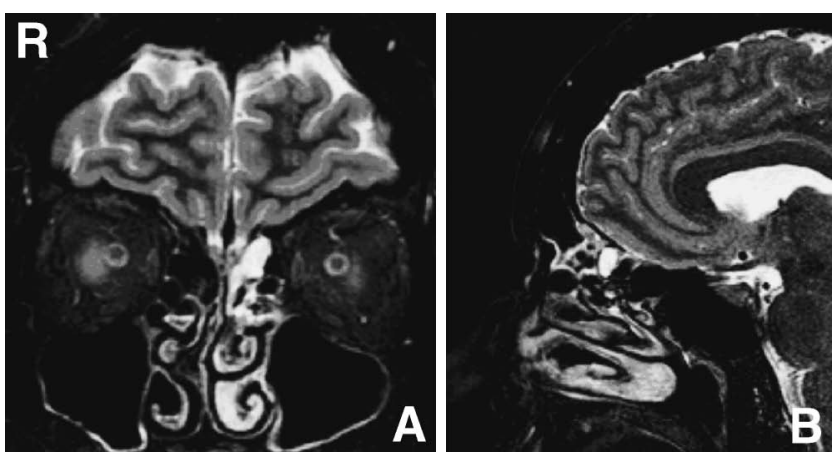

Fig. 1 Preoperative coronal (A) and sagittal (B) $T_{2}$-weighted magnetic resonance images showing cerebrospinal fluid leakage at the level of the anterior part of the ethmoidal planum, weeping into the anterior ethmoidal cell complex.

7-day period of bed rest, while prophylactic broad-spectrum antibiotic treatment and acetazolamide were administered. Despite slight improvements in headache, leftsided rhinorrhea did not resolve. High-resolution computed tomography of the facial bones was performed, revealing a small linear fracture of the left ethmoidal roof. Further investigation using maxillo-facial magnetic resonance imaging with $\mathrm{T}_{2}$-weighted fast spin-echo sequences helped to better define the fistulous tract. The point of CSF egress was identified at the level of the anterior ethmoidal planum, draining into the left anterior ethmoidal sinus (anterior ethmoidal cell complex) (Fig. 1). No damage to brain tissue was evident. The patient was referred to our hospital for surgical repair of the osteodural defect. On admission, the presence of spontaneous, continuous, left-side CSF rhinorrhea that was non-modifiable by head movement was confirmed. Results of neurological examination were otherwise unremarkable.

An endoscopic endonasal transethmoidal approach through the left nasal nostril was performed with a $0^{\circ}$, 4-mm rigid endoscope (Karl Storz GmbH \& Co. KG, Tuttlingen, Germany) as the sole visualizing tool for the whole procedure. Careful inspection of the roof of the nasal cavity, cribriform plate and fovea ethmoidalis was conducted as the first surgical step, to identify any other possible site of CSF seeping. The left middle turbinate was then removed to create a wider surgical corridor and better expose the skull base area of the defect. As the leak had been localized lateral to the middle turbinate, the uncinate process was removed to gain access to the anterior wall of the ethmoid bulla. After opening the anterior ethmoid, the CSF-leaking defect was identified at the level of the left ethmoidal roof, immediately posterior to the frontal sinus (Fig. 2A). A tiny dural tear was observed in a small bone defect. Once the fistulous tract was visualized, the reconstruction phase took place with the removal of the surrounding ethmoidal mucosa to prevent mucocele formation and to allow the graft to firmly adhere to the bony surfaces. An "overlay" technique was preferred to seal the osteodural breach: collagen matrix (Duragen ${ }^{\circledR}$; Integra LifeSciences Corp., Plainsboro, NJ, USA) was placed over the dural tear, wedging the graft under the bony edge (Fig.
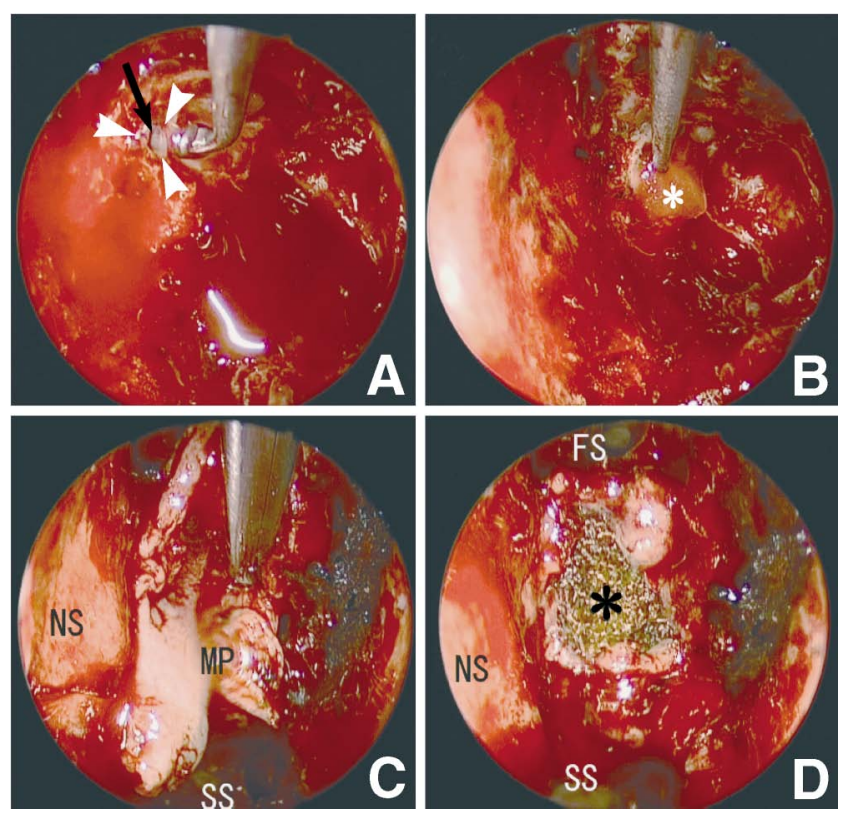

Fig. 2 Intraoperative endoscopic views showing (A) exposure of cerebrospinal fluid leaking through the osteodural defect (arrowheads), identified after removal of the ethmoidal labyrinth; (B) positioning of a collagen matrix layer over the dural tear and wedged under the bony edges (this image was taken while placing the substitute, as the substitute is extremely difficult to identify after placement); (C) covering with mucoperichondrium (MP) harvested from the removed middle turbinate; and (D) reinforcement of the duraplasty with oxidized cellulose. FS: frontal sinus, NS: nasal septum, SS: sphenoid sinus. Arrow shows the dural tear; asterisk, reconstruction materials in place at the level of the anterior roof of the ethmoid sinus.

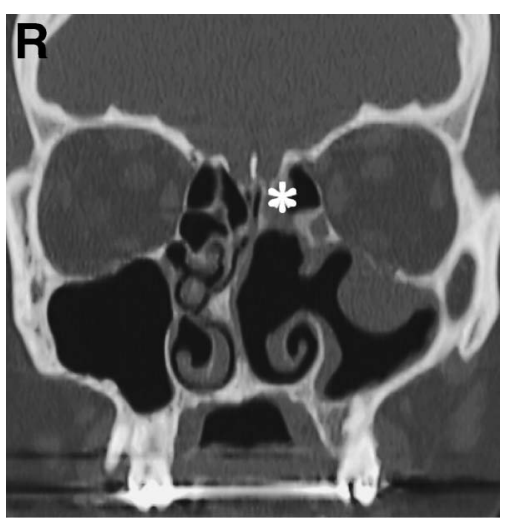

Fig. 3 Coronal computed tomography scan of the facial bones at 3 months postoperatively, showing the repaired osteodural defect (asterisk), at the level of the left anterior ethmoidal roof.

2B). The duraplasty was then reinforced by positioning oxidized cellulose (Tabotamp ${ }^{\circledR}$; Johnson \& Johnson, New Brunswick, NJ, USA), mucoperichondrium harvested from the removed middle turbinate (Fig. 2C) and a new thin fleece of collagen matrix (Fig. 2D), covered by a layer of fibrin glue. Prior to finishing the procedure, a Valsalva 
maneuver was performed to ensure the efficacy of the repair in sealing the CSF leak.

Postoperative course was uneventful. Diuretics and stool softeners were administered to reduce CSF pressure and permit rapid sealing of the reconstruction materials over the defect, and broad-spectrum antibiotics were administrated to minimize the risk of bacterial contamination. On postoperative day 3, endoscopic examination of the nasal cavities on the ward confirmed a watertight closure. The patient was then allowed to get out of bed and was discharged 2 days later. At the 3-month follow up, no clinical or radiological evidence of recurrence was identified (Fig. 3).

\section{Discussion}

Surgical management of traumatic CSF leaks was first reported by Dandy, who repaired a cranionasal fistula by means of frontal craniotomy in 1926. ${ }^{10)}$ Since then, transcranial approaches have been adopted routinely for the repair of osteodural defects, even after minor maxillofacial and/or head traumas. ${ }^{20-22)}$ A different perspective on the surgical treatment of anterior skull base fractures was not proposed until the early 1980s. The first purely endoscopic repair of a CSF leak was described in 1981.23) Over recent decades, endoscopic endonasal approaches beyond the sellar region have become more widespread, thanks to the combined contributions of both neurosurgeons and otorhinolaryngological surgeons, ${ }^{2,13,21)}$ providing the opportunity to consider this technique as a possible option for post-traumatic CSF rhinorrhea.,8,9,17-19,24) Many authors have recently reported the successful repair of osteodural defects of the anterior skull base using either a purely endoscopic or an endoscopic-assisted endonasal technique. ${ }^{1,2,7-9,12,15,17-19)}$ Nevertheless, the choice of the best surgical approach depends primarily on the etiology of the fistula, the main characteristics of the trauma and the anatomical features of the leak site. ${ }^{18)}$

Transcranial approaches are recommended for cases involving extensive dural tears, large skull base defects, multiple fractures, and associated severe brain injuries, i.e., where the likelihood of recurrence is high. ${ }^{8,21)}$ On the other hand, the endoscopic endonasal technique has been seen to accomplish better results in the repair of more anteriorly located skull base fractures, presenting round, well-defined borders, small tears of the dura mater and, eventually, absence of concomitant brain injuries. ${ }^{18,19)}$ As a matter of fact, post-traumatic CSF leaks have been found to occur more frequently in those areas of the anterior skull base where the dura mater is strictly adherent to the bone, i.e., the cribriform plate and the anterior and posterior ethmoidal roof. ${ }^{22)}$

Identification of post-traumatic CSF leakage is straightforward in those patients who present with either intermittent or persistent CSF rhinorrhea, but could be more difficult to recognize in patients with only subtle clinical symptoms, such as headache, sudden fever, and/or neck stiffness. In these latter situations, strongly suggesting the presence of a CSF fistula, provocative maneuvers such as the Valsalva maneuver and/or severe head flexion should be attempted to reveal the leakage. Furthermore, since disruption of the physiological osteodural barrier creates a direct communication between a sterile area (i.e., the intracranial compartment) and a contaminated area (i.e., the extracranial environment) through the sinonasal cavities, the occurrence of meningitis as a presenting sign seems quite common (10\%), even if it remains a dramatic event. ${ }^{18)}$

Concerning treatment options, in cases of minor CSF weeping, conservative management is often successful and includes avoidance of straining, bed rest with or without slight head elevation, and eventually, pharmacological therapy with diuretics inhibiting carbonic anhydrase and broad-spectrum prophylactic antibiotic therapy to prevent meningitis. Placement of a lumbar drain can prove very useful, diverting CSF flow away from the osteodural breach and thus reducing pressure on the breach while the defect heals.

Surgical closure must be considered if conservative treatments fail. Repair can be performed by choosing suitable synthetic, autologous or heterologous materials (e.g., turbinate mucoperichondrium, fascia lata, periumbilical fat tissue, nasoseptal mucosa, bovine or equine pericardium, collagen sponge, titanium mesh, polylactic acid) that can be used in several different methods, individually or combined in a multilayer fashion. Recently, a variety of techniques for the reconstruction of skull base defects have been reported..$^{5,7,14-18)}$

Based on more than 800 endoscopic endonasal approaches to the sellar region and skull base, we have noticed that the most effective closure method is the extradural technique. ${ }^{\text {7) }}$ Therefore, in this case we used a thin layer of synthetic dural substitute in an "overlay" position, between the dural tear and the bone,,$^{7)}$ covered by autologous mucoperichondrium harvested from the removed middle nasal turbinate, and positioned with the mucosal surface facing the sphenoidal cavity. Thereafter, another layer of collagen matrix was placed and the sphenoid sinus was packed with fibrin glue to fill the dead space, thus holding the graft in place. ${ }^{3,5-7,18)}$ In this manner, even limited incisions to the abdomen or leg to harvest fascia lata or fat tissue can be avoided, as previous reconstructions have generally been performed using abdominal fat or fascia lata. ${ }^{1,5,6,9,18)}$ Nevertheless, no consensus has yet been established regarding the material or combination of materials and methods that can be considered the optimal remedy for repairing osteodural defects, with each option providing a similar number of pros and cons. The choice of adequate technique and materials to be used should therefore be addressed in each case based on the experience of the surgeon and the features of the osteodural defect. ${ }^{5,7,18)}$ Indeed, the presence of fragmentary defects, inappropriate preparation of the surgical field and incorrect graft selection are only some of the issues reported as key reasons for repair failure.

Complete, accurate and integrated preoperative planning, mostly based on high-resolution imaging with reconstructions on coronal and sagittal planes, is warranted to better define the leak site and related anatomy, representing the backbone to obtaining successful surgical repair. Moreover, accurate knowledge of the paranasal sinus and 
anterior skull base anatomy and refined know-how for endoscopic surgery are essential to adequately perform such techniques, providing precise surgical orientation. In those cases where the fistula is particularly difficult to identify intraoperatively, the possibility of falling back on the fluorescein test should be kept in mind. ${ }^{19}$

Finally, we would like to highlight several advantages related to the endoscopic endonasal technique itself: this represents a minimally invasive approach when compared to transcranial approaches. Using the endonasal corridor allows the surgeon the opportunity to reach a more noble structure, the brain and intracranial compartment, through a less noble one. Furthermore, this method offers opportunities to obtain autologous reconstruction materials such as the mucosal nasoseptal flap and/or turbinatal mucoperichondrium, without any esthetic damage or postoperative patient discomfort.

Spontaneous rhinorrhea can sometimes represent the presenting sign of occult fracture of the skull base, even after a minor head or maxillofacial trauma. Surgical repair is recommended only for cases in which conservative treatments have failed. The aim of surgery is to achieve an effective watertight seal of the CSF leak, providing permanent dural repair. The endoscopic endonasal approach provides a success rate of $90 \%$. However, adequate endoscopic skills and specific anatomical knowledge of the paranasal sinus and, above all, of the ventral aspect of the skull base, as well as careful selection of candidate patients, are essential to obtain such excellent surgical results.

\section{References}

1) Briggs RJ, Wormald PJ: Endoscopic transnasal intradural repair of anterior skull base cerebrospinal fluid fistulae. $J$ Clin Neurosci 11: 597-599, 2004

2) Cappabianca P, Cavallo LM, Esposito F, de Divitiis O, Messina A, de Divitiis E: Extended endoscopic endonasal approach to the midline skull base: the evolving role of transsphenoidal surgery, in Pickard JD, Akalan N, Di Rocco C, Dolenc VV, Lobo Antunes J, Mooij JJA, Schramm J, Sindou $M$ (eds): Advances and Technical Standards in Neurosurgery. Wien, New York, Springer, 2008, pp 152-199

3) Cappabianca P, Esposito F, Magro F, Cavallo LM, Solari D, Stella L, de Divitiis O: Natura abhorret a vacuo-use of fibrin glue as a filler and sealant in neurosurgical "dead spaces". Technical note. Acta Neurochir (Wien) 152: 897-904, 2010

4) Cappabianca P, Frank G, Pasquini E, de Divitiis O, Calbucci F: Extended endoscopic endonasal transsphenoidal approaches to the suprasellar region, planum sphenoidale and clivus, in de Divitiis E, Cappabianca P (eds): Endoscopic Endonasal Transsphenoidal Surgery. Wien, New York, Springer, 2003, pp 176-187

5) Castelnuovo P, Locatelli D, Mauri S: Extended endoscopic approaches to the skull base. Anterior cranial base CSF leaks, in de Divitiis E, Cappabianca P (eds): Endoscopic Endonasal Transsphenoidal Surgery. Wien, New York, Springer, 2003, pp 137-158

6) Castelnuovo P, Mauri S, Locatelli D, Emanuelli E, Delu G, Giulio GD: Endoscopic repair of cerebrospinal fluid rhinorrhea: learning from our failures. Am J Rhinol 15: 333-342, 2001

7) Cavallo LM, Messina A, Esposito F, de Divitiis O, Dal Fabbro M, de Divitiis E, Cappabianca P: Skull base reconstruc- tion in the extended endoscopic transsphenoidal approach for suprasellar lesions. J Neurosurg 107: 713-720, 2007

8) Chin GY, Rice DH: Transnasal endoscopic closure of cerebrospinal fluid leaks. Laryngoscope 113: 136-138, 2003

9) Cukurova I, Cetinkaya EA, Aslan IB, Ozkul D: Endonasal endoscopic repair of ethmoid roof cerebrospinal fluid fistula by suturing the dura. Acta Neurochir (Wien) 150: 897-900, 2008

10) Dandy WD: Pneumocephalus. Arch Surg 12: 949-982, 1926

11) de Divitiis E, Cavallo LM, Cappabianca P, Esposito F: Extended endoscopic endonasal transsphenoidal approach for the removal of suprasellar tumors: Part 2. Neurosurgery 60: 46-59, 2007

12) Gardner P, Kassam A, Snyderman C, Mintz A, Carrau R, Moossy JJ: Endoscopic endonasal suturing of dural reconstruction grafts: a novel application of the U-Clip technology. Technical note. J Neurosurg 108: 395-400, 2008

13) Jho HD, Carrau RL: Endoscopic endonasal transsphenoidal surgery: experience with 50 patients. J Neurosurg 87: 44-51, 1997

14) Kassam A, Carrau RL, Snyderman CH, Gardner P, Mintz A: Evolution of reconstructive techniques following endoscopic expanded endonasal approaches. Neurosurg Focus 19(1): E8, 2005

15) Kassam AB, Thomas A, Carrau RL, Snyderman CH, Vescan A, Prevedello D, Mintz A, Gardner P: Endoscopic reconstruction of the cranial base using a pedicled nasoseptal flap. Neurosurgery 63(1 Suppl 1): ONS44-ONS53, 2008

16) Kitano $M$, Taneda $M$ : Subdural patch graft technique for watertight closure of large dural defects in extended transsphenoidal surgery. Neurosurgery 54: 653-661, 2004

17) Lee TJ, Huang CC, Chuang CC, Huang SF: Transnasal endoscopic repair of cerebrospinal fluid rhinorrhea and skull base defect: ten-year experience. Laryngoscope 114: 14751481, 2004

18) Locatelli D, Rampa F, Acchiardi I, Bignami M, De Bernardi F, Castelnuovo P: Endoscopic endonasal approaches for repair of cerebrospinal fluid leaks: nine-year experience. Neurosurgery 58(4 Suppl 2): ONS-246-ONS-257, 2006

19) Nachtigal D, Frenkiel S, Yoskovitch A, Mohr G: Endoscopic repair of cerebrospinal fluid rhinorrhea: is it the treatment of choice? J Otolaryngol 28: 129-133, 1999

20) Nohra G, Jabbour P, Haddad A, Abouhamad W, Abilahoud G, Okais N: [Subcranial subfrontal approach for the treatment of extensive cerebrospinal fluid leaks]. Neurochirurgie 48(2-3 Pt1): 87-91, 2002 (French)

21) Samii M, Draf W: [Indication and treatment of frontobasal rhinoliquorrhoea from the ent-surgical and neurosurgical point of view (author's transl)]. Laryngol Rhinol Otol (Stuttg) 57: 689-697, 1978 (German)

22) Scholsem M, Scholtes F, Collignon F, Robe P, Dubuisson A, Kaschten B, Lenelle J, Martin D: Surgical management of anterior cranial base fractures with cerebrospinal fluid fistulae: a single-institution experience. Neurosurgery 62: 463471, 2008

23) Wigand ME: Transnasal ethmoidectomy under endoscopical control. Rhinology 19: 7-15, 1981

24) Zweig JL, Carrau RL, Celin SE, Schaitkin BM, Pollice PA, Snyderman CH, Kassam A, Hegazy H: Endoscopic repair of cerebrospinal fluid leaks to the sinonasal tract: predictors of success. Otolaryngol Head Neck Surg 123: 195-201, 2000

Address reprint requests to: Mika Komatsu, MD, Department of Neurosurgery, Fukuoka University Faculty of Medicine, 7-45-1 Nanakuma, Johnan-ku, Fukuoka 814-0180, Japan. e-mail: approachgogo@yahoo.co.jp 